\title{
A bi-objective home health care routing and scheduling model with considering nurse downgrading costs
}

\author{
Pouria Khodabandeh", Vahid Kayvanfar', Majid Rafiee', and Frank Werner,** \\ 1 Department of Industrial Engineering, Sharif University of Technology, Tehran, Iran; \\ pouriakhodabandeh@ie.sharif.edu (P.Kh.); kayvanfar@sharif.edu (V.K); rafiee@sharif.edu (M.R). \\ 2 Faculty of Mathematics, Otto-von-Guericke-University, Magdeburg, Germany; e-mail@e-mail.com \\ * Correspondence: frank.werner@mathematik.uni-magdeburg.de
}

\begin{abstract}
In recent years, the management of health systems is a main concern of governments and decision makers. Home health care is one of the newest methods of providing services to patients in developed societies that can respond to the individual lifestyle of modern age and the increase of life expectancy. The home health care routing and scheduling problem is a generalized version of the vehicle routing problem, which is extended to a complex problem by adding special features and constraints of health care problems. In this problem, there are multiple stakeholders such as nurses for which an increase of their satisfaction level is very important. In this study, a mathematical model is developed to expand traditional home health care routing and scheduling models to downgrading cost aspects by adding the objective of minimizing the difference between the actual and potential skills of the nurses. Downgrading can lead to a dissatisfaction of the nurses. In addition, skillful nurses have higher salaries and high-level services increase equipment costs and need more expensive trainings and nursing certificates. Therefore, downgrading can enforce hidden huge costs to the managers of a company. To solve the bi-objective model, an -constraint based approach is suggested and the model applicability and its ability to solve the problem in various sizes are discussed. A sensitivity analysis on the Epsilon parameter is conducted to analyze the effect of this parameter on the problem. Finally, some managerial insights are presented to help the managers in this field, and some directions for future studies are mentioned as well.
\end{abstract}

Keywords: Home health care; Routing and scheduling; Nurse downgrading; Epsilon-constraint method; Bi-objective optimization.

\section{Introduction}

Today one of the main concerns of policymakers in different societies is the proper management of health systems. These systems, in addition to having a significant impact on public health, impose significant costs on the society. Also, with decreasing the birth rate and increasing elderly population and the rise of chronic diseases, there is a growing need for health services, as countries have to spend a significant portion of their existing budget on the health area. On the other hand, due to resource constraints, planning for an optimal use of the resources seems to be necessary. Furthermore, in many countries, as family members become busier and far away from each other, we see the pattern of human life moving towards an individual life, especially the elderlies, which creates the need of paying special attention to this group of people more than ever before. Therefore, one of the most effective ways to reduce the use of hospital beds and clinics is to serve patients at their place of residence. One of the most important solutions to address this problem is to establish a system for delivering efficient health care at home. Home Health Care (HHC) is a wide range of health care services that can be provided in the patient's home due to illness, wound or old age. These services are usually cheaper and more convenient than those 
provided in the hospital, while being as efficient as the services provided in the hospital (Alodhayani, 2017). HHC services can be seen as an essential complement to health care in developed countries (Tyan, 2010). In this context, home service providers are often confronted with contradictions in their goals as they aim to minimize operating costs while they wish to maximize the level of offering customer service. One of the related problems that costs a lot for the companies is the optimal routing and scheduling for service providers, which has received much attention in recent years. The Home Health Care Routing and Scheduling Problem (HHCRSP) can be described as a dispersed collection of patients in a geographic area who require home health care, which must be provided by nurses. The HHCRSP includes designing a set of routes to deliver the scheduled care services within a planning horizon that minimizes criteria such as cost or maximizes the service quality by taking into account a number of constraints.

The problem of routing and scheduling of HHC services was first proposed by Fernandez et al. (1974). Bertels and Fahle (2006) as well as Eveborn et al. (2006) presented optimization methods embedded in Decision Support Systems (DSS). Bertels and Fahle introduced a meta-heuristic approach, and Eveborn et al. introduced a heuristic method and a recurrent matching algorithm for solving the problem. Akjiratikarl et al. (2007) modeled the problem as a Vehicle Routing Problem (VRP) and used the PSO meta-heuristic optimization method. Time window, required skills, and working time rules are common factors in most HHC routing and scheduling problems. However, the specific applications of these constraints between different papers have substantially differences with each other. In the context of when services should start, most authors have considered a hard type of a time window. In addition, the soft time window can be accessed in a wide range of papers to respect the preferences of the patient, such as Bertels and Fahle (2006), Eveborn et al. (2006), Trautsamwieser et al. (2011), Trautsamwieser and Hirsch (2011), Mankowska et al. (2014), Misir et al. (2015), Yuan et al. (2015), Braekers et al. (2016). Furthermore, several papers considered a time window for each nurse (e.g., Dohn et al. (2009), Allaoua et al. (2013), Hiermann et al. (2015), Mutingi and Mbohwa (2014)) which determines when a specific nurse could offer a service to patients. In addition, matching the skills of the nurses and the needs of the patients is a common feature in HHC optimization, and the domain of the skills considered may vary depending on the needs of the patients and a specific set of rules. However, in Bertels and Fahle (2006), additional and non-compulsory skills are also considered as soft constraints. For example, balancing the distribution of difficult visits among all nurses is considered. In some cases, downgrading has been permitted (see e.g. Trautsamwieser et al. (2011), Trautsamwieser and Hirsch (2011), Hiermann et al. (2015), Rest and Hirsch (2016). This means that a higher skilled nurse can provide a lower level service. While it provides a better flexibility in the planning process and reduces travel costs, the company incurs higher costs for higher skilled nurses. On the other hand, it can lead to a dissatisfaction of highly skilled nurses. However, in these previous studies the huge effect of downgrading costs on an optimal planning of the HHCRSP and the role of the decision maker were not considered. Home health care services are expensive and company managers should decide on their acceptable downgrading level and plan their operations by considering these important downgrading cost aspects. Another issue that is considered in home health care is hourly labor law, which usually determines 5 to 10 hours a day or a time window is considered. Several authors have taken a time window preference and calculated the violation by means of a penalty (see for example, Trautsamwieser et al. (2011), Trautsamwieser \& Hirsch (2011), Fikar \& Hirsch (2015)). Since most papers consider the HHC routing and scheduling problem as an extension of the VRP, the main focus is on travelling. However, unlike the classic VRPs where travel distances are minimized (see for example Toth \& Vigo (2014)), in the HHC problems, the focus is often on travel costs, travel time, and the working time of the nurses. For this reason, most of the works involve overtime and waiting time (see e.g., Trautsamwieser et al. (2011), Trautsamwieser \& Hirsch (2011), Hierman et al. (2015), Misir et al. (2015)). Only a few studies explicitly minimize the number of nurses at the start of the route (e.g., Allaoua et al. (2013), Yuan et al. (2015). Donh et al. (2009) proposed a framework based on a branch and price (B\&P) algorithm for scheduling concurrent tasks. They considered HHC as a practical area that could use branch and price, but they used examples from airport operations for the computational experiments. Redjem and Marcon (2016) used heuristic solution methods to 
manage real-size samples, they offered a two-step heuristic approach that continuously shifts jobs to meet the time constraints. Rodriguez et al. (2015) considered staff dimensioning aspects of home health care in a tactical horizon to ensure that the HHC company can meet its required tasks. Spatial dimensions and a combination of the nurse skills increased the complexity of their problem. In their study, demands are non-deterministic, and a two-stage integer stochastic approach is proposed. Their algorithm can give the number of nurses needed from each category without any overtime cost or external resources. Liu et al. (2016) proposed a mathematical model with the consideration of lunch break requirements and decomposed it into a master problem and several pricing sub-problems. They used a branch-and-price algorithm (B\&P) to solve the problem. In their solution approach, a label-correcting algorithm is applied to the lunch break constraints and in the column generation process, some acceleration strategies are used, as well. Yuan et al. (2018) proposed a daily HHCRSP with considering non-deterministic travel and service times. These assumptions are derived from possible changes in the patient health status and road traffic conditions that are valid in the practical world of HHC. First, they used stochastic programming with recourse, where the recourse action is to skip patients without services if the nurse arrives later than their latest starting service time. Then, a set partitioning model is proposed and a branch-and-price algorithm is used for solving the problem. Liu et al. (2018) presented a bi-objective model to minimize the company costs and, on the other hand, to improve patient satisfaction. Decerle et al. (2018) highlighted the multi-objective home health care problem with the centrality of practical planning and applied a memetic algorithm to solve it. Nasir and Dang (2018) extended the conventional HHCRSP to capacity and demand management aspects. To handle this problem, they proposed a mixed integer programming (MIP) model considering workload balancing, and then a heuristic method as well as a variable neighborhood search (VNS) algorithm were applied to solve it. Nasir et al. (2018) presented a mathematical model so as to integrate resource dimensioning issues and assignment aspects considering the telehealth-based care and patients' group-based care services. Fathollahi-Fard et al. (2018) presented a bi-objective green home health care model that addresses environmental pollution. Decerle et al. (2019) presented an algorithm combining memetic and ant colony optimization techniques that took into account synchronization, workload balance and time windows.

As one can see from the reviewed literature and to the best of the authors' knowledge, most of the researches did not pay any attention to downgrading cost concepts as an important home health care aspect. In some previous researches, some nurses with high qualification levels were permitted to provide some usual and low level services to patients. But in these studies, downgrading concepts were not considered from the top-level home health care managers' point of view and their huge downgrading costs that are enforced to their company in each day. In the real world of the home health care industry, there are various nurse skills that are very expensive and taking their nursing certificate is complex and time-consuming. So the companies should configure their plans to use most of the potential qualifications of their nurses. In this way, they reduce their hidden costs and increase the satisfaction level of the nurses. In this study, such requirements led us to develop conventional models to a bi-objective novel model which can engage downgrading costs into the home health care routing and scheduling problem. The managers of a home health care company can make a tradeoff between the total nurse traveling times and the downgrading costs that are very costly and important for the management of home health care human resources. An Epsilon-constraint based solution approach is presented to handle this bi-objective optimization problem. The main purpose of this approach is to provide feasible and even optimal solutions for the decision maker. The decision maker can adjust different values of the Epsilon parameter to analyze the effects of various downgrading levels on the objective concerning the total traveling times of the nurses and the whole planned routes.

The rest of this paper is organized as follows. Section 2 describes the problem and the mathematical model. Section 3 discusses the solution approach. Experimental results and a sensitivity analysis are presented in Section 4 . Section 5 suggests some managerial insights and finally, some conclusions and future studies are briefly presented in Section 6 . 


\section{Problem Description}

In general home health care routing and scheduling studies, researchers have been always trying to enhance the quality of services which are provided to patients while reducing the costs of the service provider. In addition to the main goals of this problem, nurse satisfaction is one of the most important and common concerns of the companies. Ignoring nurse satisfaction can cause huge hidden costs for the companies.

In this problem, each of the nurses has different skill levels. The best situation for assigning nurses to patients is to use all their skills. If the company does not use some of the skills of the nurses, this could lead to nurse dissatisfaction, and this situation is called downgrading. In fact, the downgrading concept is the difference between the potential skills of the nurses and the actual skills that are used in the planning process. In addition to nurse dissatisfaction, downgrading can enforce huge hidden costs to the company. Nurses with different qualifications and skill levels have different salaries and other associated equipment costs. Thus, when some available manpower capacity of the company is not used, some additional huge costs are compelled to the company.

By investigating the previous researches in this area, efforts of decreasing the difference gap between the potential skills of the nurses and their actual planned services as an optimization goal have not been observed. Therefore, in this study, the necessity of matching the potential skills of the nurses with their used skills is addressed, and a novel mathematical model is presented that aims to reduce the downgrading costs of the nurses along with reducing the total traveling time of the nurses. Home health care companies are incurred different downgrading costs by ignoring various nurse qualifications. Therefore, the value of each service type is considered as different, and is determined by the planning's decision maker. The weighted values of various nurse service types are considered by the parameter $w_{\mathrm{s}}$. In this model, each patient requires a variety of services, where all of them must be answered by qualified nurses. Because of the sensitivity and importance of a patient's health condition, all patients should be served in their optimal time window, and the sequence of services provided by the nurses should be respected. In addition, HHCRSP is an extended VRP problem, so it is important to consider the specific features of the VRP problem in this study. In this model, the starting and ending point of each nurse is the depot, and each nurse should depart from the patient's place after serving him/her.

The planning of the new model presented in this study allows the decision maker to establish a balance between a reduction of the traveling costs of the nurses in exchange for the hidden costs of not fully utilizing the skills of the nurses.

The rest of this part is organized as follows. In Section 2.1, the model assumptions are described. In Section 2.2, the model notations are introduced, and the mathematical model is presented in Section 2.3.

\subsection{Assumptions}

- $\quad$ Several different service needs and qualifications are included into the problem.

- All service needs of patients should be provided by qualified nurses.

- Each route of a nurse is started from the depot.

- Each nurse should end its path at the depot after visiting all planned patients.

- Each patient's acceptable time window should be respected.

- The parameters of the patient demand, traveling time and service time are known before the planning and considered to be deterministic.

- The correct servicing sequence of each nurse should be respected by considering the service time of the previous patient in addition to the time needed for traveling between the patients' places.

- A single period planning strategy is considered in the problem.

- Travel sharing and multi-mode traveling concepts are not considered.

- Emergent situations and urgent service needs are not included into the problem.

\subsection{Notations}




\subsubsection{Subscripts}

$i \quad$ Starting node index of each travel $(i=1,2, \ldots, n+1)$, where $n$ denotes the number of patients in planning.

$j \quad$ Ending node index of each travel $(j=2,3, \ldots n+2)$, where $n$ denotes the number of patients in planning.

$k \quad$ Index for the nurses $(k=1,2, \ldots, V)$, where $V$ denotes the number of nurses in planning.

$s \quad$ Index for the services $(s=1,2, \ldots, S)$, where $S$ denotes the number of different services in the planning.

\subsubsection{Sets}

C Set of patients.

$N \quad$ Set of all nodes that includes patients and the depot.

$V \quad$ Set of nurses.

$S \quad$ Set of services.

\subsubsection{Input Parameters}

$t_{i j} \quad$ Travel time between node $i$ and node $j$.

$t_{i s} \quad$ Required time for offering service $s$ to patient $i$.

$l_{i} \quad$ Lower bound on the patient time window.

$u_{i} \quad$ Upper bound on the patient time window.

$\mathrm{a}_{\mathrm{ks}}$ Input matrix of nurse qualifications, where 1 means that nurse $k$ has the qualification of doing service $s$.

$g_{\text {js }} \quad$ Input matrix of patient's service needs, where 1 means that patient $j$ needs service $s$.

$w_{\mathrm{s}} \quad$ Weighted value of service $s$ for the decision maker.

$\varepsilon^{\prime} \quad$ A small positive number, e.g. 0.1.

\subsection{Decision Variables}

$x_{i j k s} 1$ if nurse $\mathrm{k}$ transfers from node $i$ to $\mathrm{j}$ for offering service $s ; 0$ otherwise.

$S_{i k s}$ Starting time of offering service $s$ to patient $i$ by nurse $k$.

$b_{k s} \quad 1$ if service $s$ of nurse $k$ is used in the optimal planning; 0 otherwise.

\subsection{The mathematical model}

\subsubsection{Objective function}

$$
\begin{aligned}
& f 1: \text { Min } z=\sum_{i \in N} \sum_{j \in N} \sum_{k \in V} \sum_{s \in S} t_{i j} x_{i j k s} \\
& f 2: \operatorname{Min} z=\sum_{k \in V}\left(\sum_{s \in S} w_{s} a_{k s}-\sum_{s \in S} w_{s} b_{k s}\right)
\end{aligned}
$$


The first objective function is stated in Eq. (1) and minimizes the total traveling time of all routes of the nurses, and the second objective function that minimizes the downgrading costs of the nurses, is presented in Eq. (2).

\subsubsection{Constraints}

$$
\begin{array}{ll}
\sum_{i \in C} \sum_{s \in S} x_{1 i k s}=1 & \forall k \in V \\
\sum_{i \in C} \sum_{s \in S} x_{i 1 k s}=1 & \forall k \in V
\end{array}
$$

Constraints (3) and (4) are used to guarantee that the starting and ending place of each nurse is the depot.

$$
\sum_{i \in N} \sum_{s \in S} x_{i j k s}-\sum_{i \in N} \sum_{s \in S} x_{j i k s}=0 \quad \forall j \in C, k \in V
$$

Constraint (5) ensures that each nurse should depart from the patient's place after giving care and go to another patient's home.

$$
S_{i k s_{1}}+t_{i s_{1}}+t_{i j}-M\left(1-x_{i j k s_{2}}\right) \leq S_{j k s_{2}} \quad \forall i, j \in N, k \in V, s_{1} \in S, s_{2} \in S
$$

Constraint (6) states that a new service should be started after the time of finishing the previous service in addition to the required time for transferring the nurse to the new place.

$$
l_{i} \leq S_{i k s} \leq u_{i} \quad \forall i \in C, k \in V, s \in S
$$

Constraint (7) indicates that each patient has an acceptable time window and that the starting time of a patient's service should be between the minimum and maximum of this time window.

$$
\sum_{k \in V} \sum_{i \in N} a_{k s} x_{i j k s}=g_{j s} \quad \forall j \in C, s \in S
$$

Constraint (8) is used to guarantee that, if patient $\mathrm{j}$ requires service $\mathrm{s}$, exactly one of the nurses with the required qualifications should go to the patient's place and give him/her service $s$.

$$
x_{i j k s}=a_{k s} \cdot g_{j s} \quad \forall i, j \in N, k \in V, s \in S
$$

Constraint (9) ensures that for giving service $s$ by nurse $k$ to patient $j$, the nurse must have the qualifications of service $s$ and the given patient must need this service.

$$
\varepsilon^{\prime} \cdot \sum_{i \in N} \sum_{j \in N} x_{i j k s} \leq b_{k s} \leq \sum_{i \in N} \sum_{j \in N} x_{i j k s} \quad \forall k \in V, s \in S
$$

Constraint (10) states that, if service $s$ of nurse $k$ is used at least one time in the planning, the decision variable $b_{k s}$ will be one and otherwise, it will be zero.

$$
b_{k s} \leq a_{k s} \quad \forall k \in V, s \in S
$$

Constraint (11) indicates that, if the nurse $\mathrm{k}$ does not have the qualification of service $\mathrm{s}$, the variable $b_{k s}$ must get the value zero. 


$$
x_{i j k s}, b_{k s} \in\{0,1\} ; S_{i k s} \in \text { int }^{+} ; i, j \in N ; k \in V ; s \in S
$$

The domain of the decision variables of the problem is defined in Condition (12).

\section{Solution Approach}

\subsection{Background}

In real-world problems, the decision maker is always confronted with conflicting goals to make his/her decision. In the home health care routing and scheduling problem as a practical problem, the decision maker tries to make the best possible decision by balancing the goals.

The Epsilon-constraint approach is one of the most popular methods of multi-objective optimization, which attempts to optimize the most important goal by considering upper or lower limit values for the other goals. In fact, in this method the main goal is considered as the objective function and the other goals are added to the constraints of the model. Various elements of the Pareto front can be obtained by a systematic variation of the constraint bounds. The basic bi-objective Epsilon-constraint method is presented in Figure 1 (Laumanns et al., 2006).

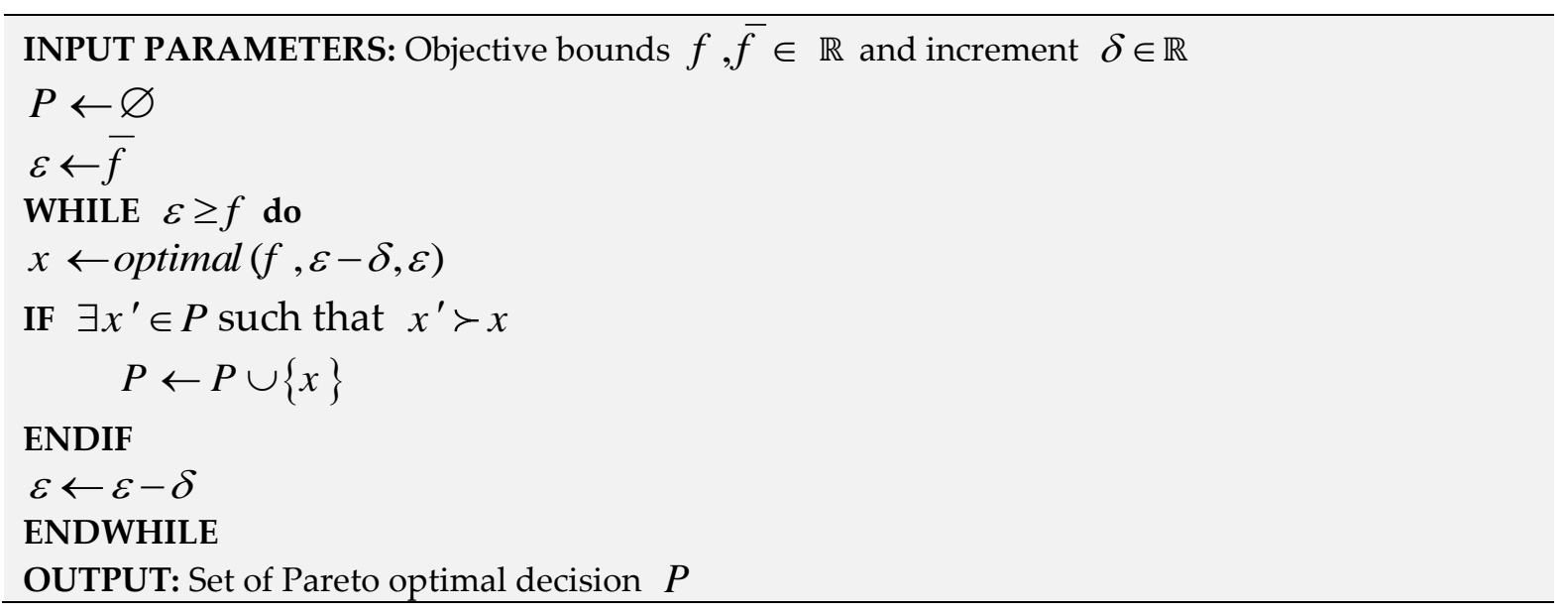

Figure 1: Pseudocode of the basic Epsilon-constraint method

\subsection{Proposed solution approach}

In this study, a bi-objective optimization method is proposed to make a tradeoff between the reduction of the traveling time of the nurses and the reduction of the downgrading costs of the nurses. This method allows the decision maker to increase the traveling time of nurses in return for reducing the downgrading costs. This solution approach is only used to obtain correct answers with considering different objectives and complex constraints of this model. Since this model is the first to consider some assumptions and HHC aspects, a comparison of these results with previous results is not possible.

In this study, the reduction of the total traveling time of the nurses is considered as the main objective function of the problem, and the second objective function that reduces the downgrading costs, is contained in the constraints by considering an upper limit specified by the Epsilon parameter. In fact, the second objective function of the model is added to the constraints with an upper bound on Epsilon and is stated as Eq. (13).

$\sum_{k \in V}\left(\sum_{s \in S} w_{s} a_{k s}-\sum_{s \in S} w_{s} b_{k s}\right) \leq \varepsilon$

The smallest amount of the Epsilon parameter can be obtained when all the skills of the nurses are used in planning, and in this case it is zero. On the other hand, if none of the skills of the nurses are used, this value is equal to the sum of the weighted value of the available skills, but this situation 
is impossible. In fact, the upper and lower limits of Epsilon would be the following values that are stated in Eq. (14).

$$
\varepsilon_{\text {lower }}=0, \varepsilon_{\text {upper }}=\sum_{s \in S} w_{s} a_{k s}
$$

To analyze the effect of changes of the Epsilon parameter on the main objective function of the problem, a heuristic approach is proposed in Figure 2 to determine the logical values of Epsilon. In this pseudo-code, the $\delta$ parameter is the reduction step of the Epsilon parameter which is determined by the decision maker.

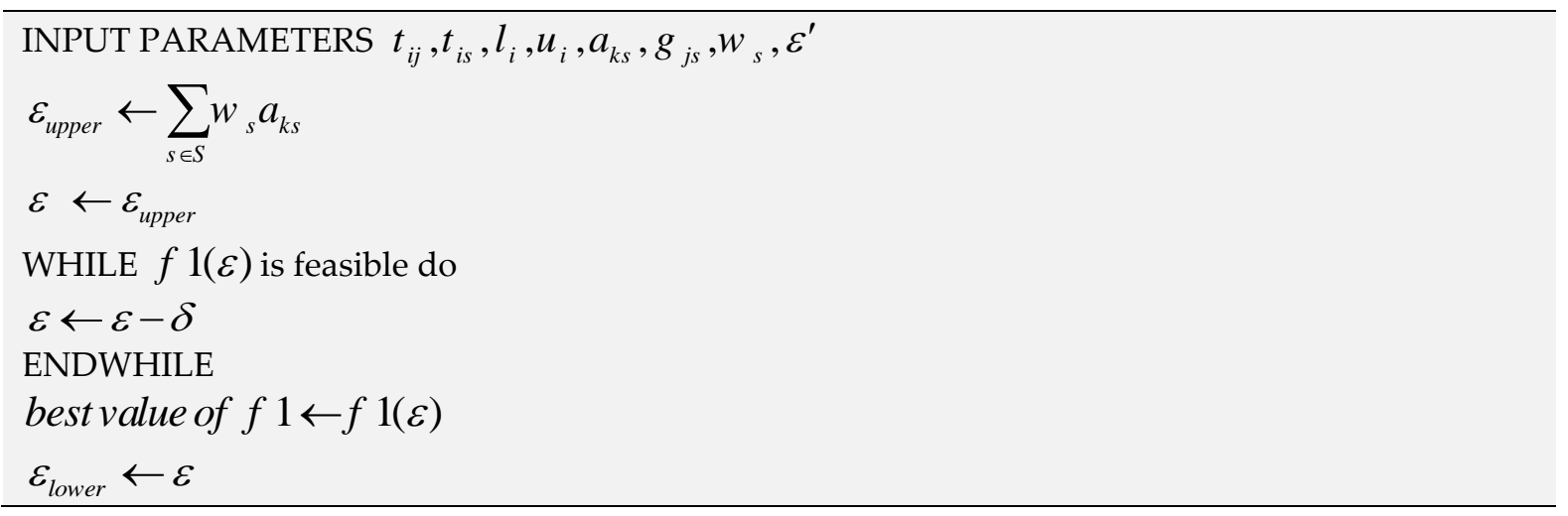

Figure 2: Pseudocode of Epsilon constraint-based approach

\section{Computational Experiments}

In this study, in order to illustrate the effectiveness of the proposed model in the real world, the model is first tested on a small example and the results are described accompanied by a discussion regarding the correctness of the suggested model. Next, the model is applied to some benchmark instances in the literature taken from Mankowska et al. (2014), and it is solved by IBM ILOG CPLEX Optimization Studio Version 12.6.0.0. All experiments in this study are run on a computer with an Intel i7-4710HQ processor, $2.5 \mathrm{GHz}$ core speed and $8 \mathrm{~GB}$ of RAM.

\subsection{Planning process}

In this subsection, a small instance is first solved to show the process of the proposed model. By doing so, the benefits of this model in contrast to traditional home health care routing and scheduling models are clarified. Table 1 shows the properties of the solved small example.

Table 1. Properties of the considered small example

\begin{tabular}{ccc}
\hline Number of patients & Number of nurses & Number of services \\
\hline 10 & 3 & 6 \\
\hline
\end{tabular}

In this small example, 10 patients and 3 nurses are considered. The patients have different service needs, and the nurses have various qualifications. There are 6 different services and 
qualifications in this problem which are presented in Table 2. Each service has a weighted value for the decision maker. In this study, the weighted values are supposed to be between 1 and 6 .

Table 2. Different service types in the small example

\begin{tabular}{c|c|c}
\hline Service ID & Service Type & Supposed weighted value \\
\hline S1 & Speech therapy & 2 \\
\hline S2 & Wound dressing & 3 \\
\hline S3 & Insulin injection & 4 \\
\hline S4 & Blood sampling & 5 \\
\hline S5 & Physiotherapy & 6 \\
\hline S6 & X-ray imaging & \\
\hline
\end{tabular}

After solving this small example by using the proposed model, the results are shown in Table 3. In this table, the routes are illustrated by arrows and each service that is given to each patient, which is shown at the top of the patient number. In this example, the parameter Epsilon is supposed to be 10.

Table 3. Optimal route of small example (Epsilon=10)

\begin{tabular}{|c|c|c|c|c|c|c|c|c|c|c|c|c|c|}
\hline \multirow[b]{2}{*}{ Nurse1 } & \multirow[b]{2}{*}{ Depot } & \multicolumn{3}{|c|}{ S5 } & \multicolumn{2}{|l|}{ S1 } & \multicolumn{2}{|l|}{ S3 } & \multirow[b]{2}{*}{ Depot } & & & & \\
\hline & & $\rightarrow$ & 8 & $\rightarrow$ & 9 & $\rightarrow$ & 5 & $\rightarrow$ & & & & & \\
\hline \multirow{3}{*}{ Nurse2 } & & & S6 & & S5 & & S3 & & S5 & & S3 & & \\
\hline & Depot & $\rightarrow$ & 8 & $\rightarrow$ & 6 & $\rightarrow$ & 10 & $\rightarrow$ & 2 & $\rightarrow$ & 7 & $\rightarrow$ & Depot \\
\hline & & & S6 & & S2 & & S4 & & $\mathrm{S} 4$ & & S4 & & \\
\hline Nurse3 & Depot & $\rightarrow$ & 10 & $\rightarrow$ & 3 & $\rightarrow$ & 1 & $\rightarrow$ & 9 & $\rightarrow$ & 4 & $\rightarrow$ & Depot \\
\hline
\end{tabular}

Next, a comparison between the results for two different Epsilon values is made. The results are illustrated in Figure 3 and Figure 4 for a better understanding. 


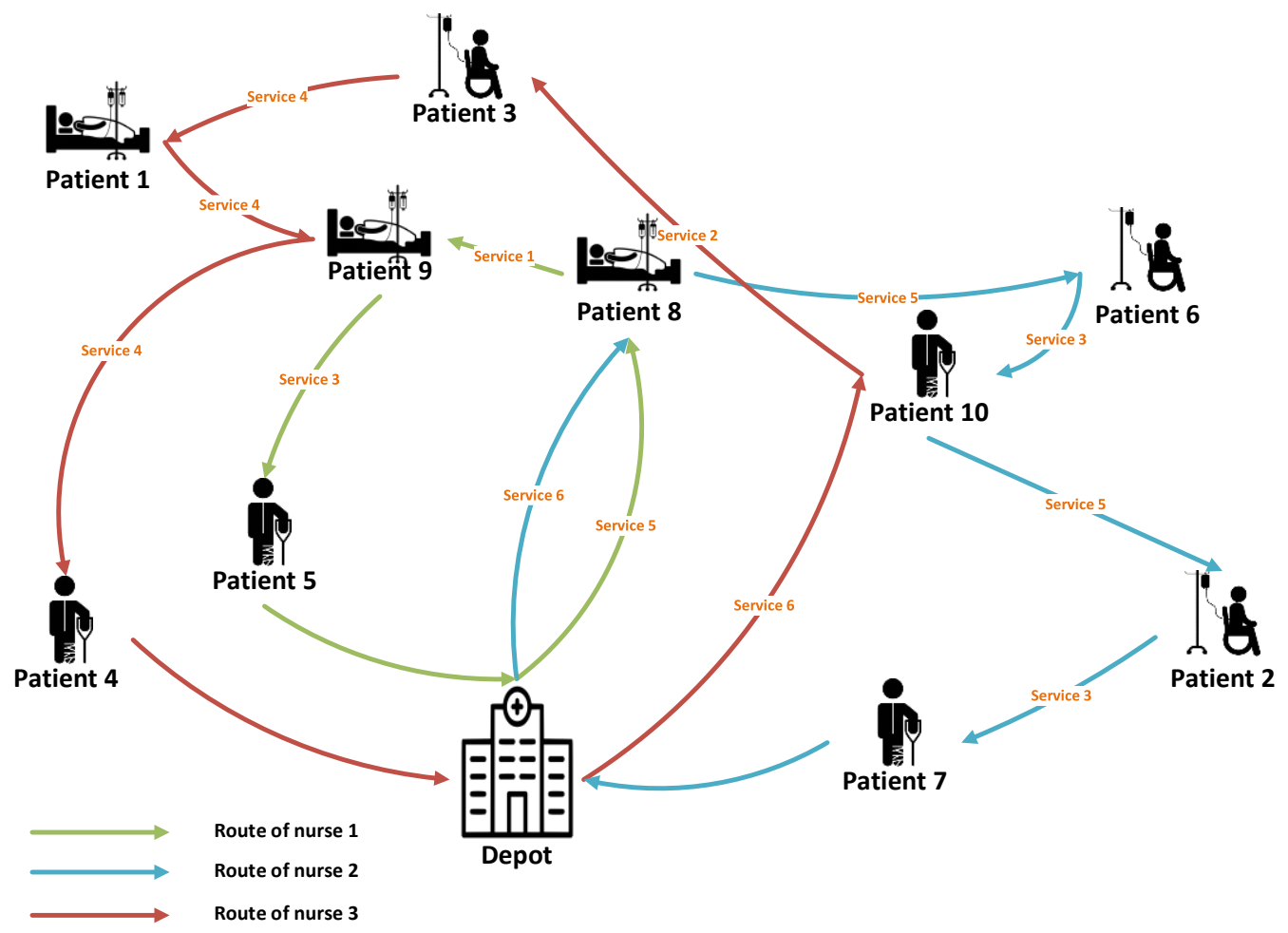

Figure 3: Optimal route of small example (Epsilon=10)

As one can see from Figure 3, each patient's service requirement is ensured and each nurse only gives services for which he/she has the qualification. In this optimal plan, some skills of the nurses can be ignored up to the downgrading level. The ignored service qualifications are presented in Table 4, where the total weighted value of them is less than the considered Epsilon value.

Table 4: Ignored service qualifications of nurses in the small example (Epsilon=10)

\begin{tabular}{c|c|c}
\hline Nurse number & Service type number & Supposed weighted value \\
\hline$\# 1$ & $\# 2$ & 2 \\
\hline$\# 2$ & $\# 1$ & 1 \\
\hline$\# 3$ & $\# 5$ & 5 \\
\hline \multicolumn{2}{r|}{ Total weighted value } \\
\hline
\end{tabular}




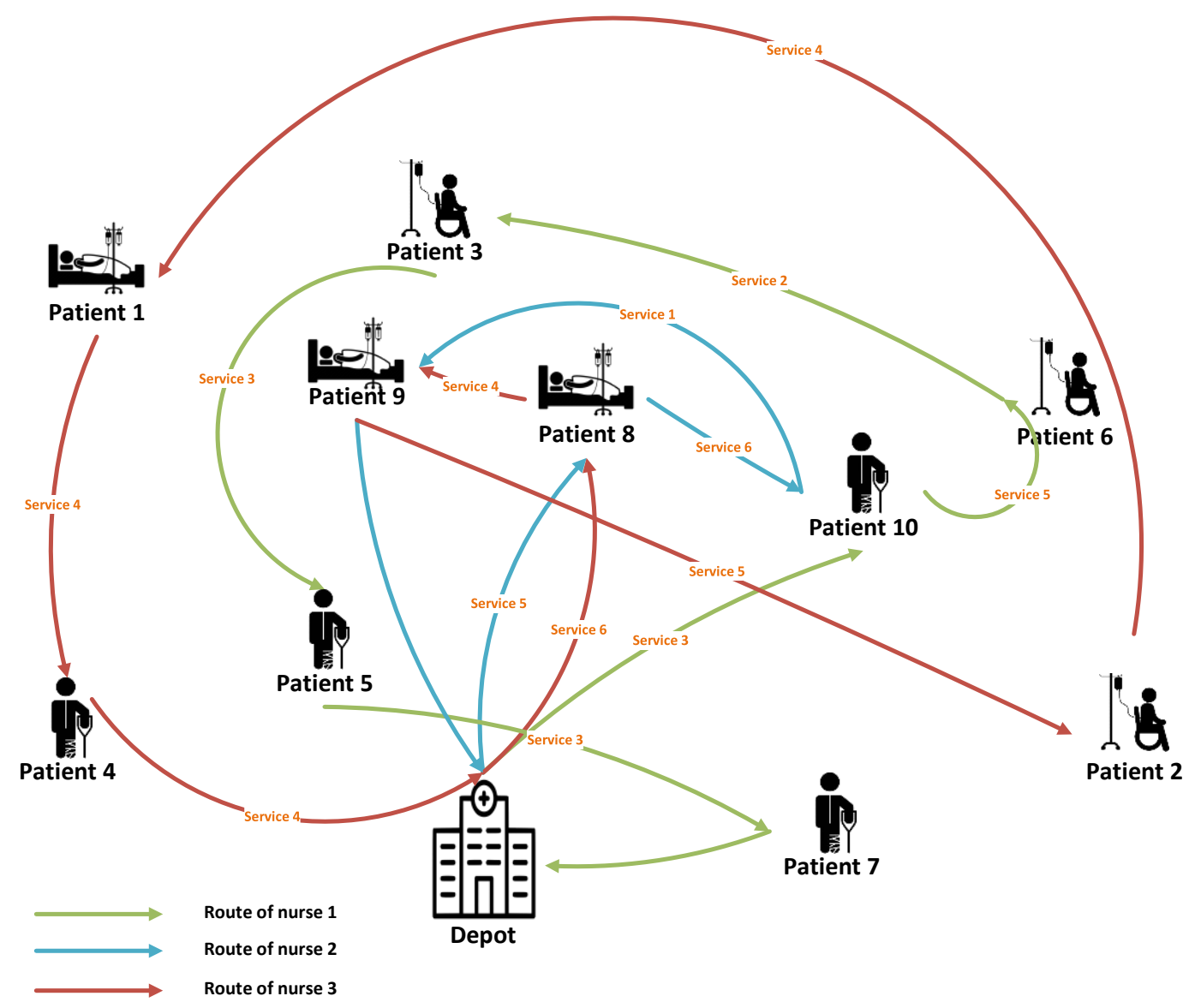

Figure 4: Optimal route of small example (Epsilon=7)

In Figure 4, with decreasing the Epsilon parameter, the whole optimal routing and scheduling is affected. However, as for the previous plan, all patients' service needs are satisfied. The ignored service qualifications of this plan are shown in Table 5, where the total weighted value of them is less than the considered Epsilon value.

Table 5: Ignored service qualifications of nurses in small example (Epsilon=7)

\begin{tabular}{c|c|c}
\hline Nurse number & Service type number & Supposed weighted value \\
\hline$\# 1$ & $\# 1$ & 1 \\
\hline$\# 2$ & $\# 3$ & 3 \\
\hline$\# 3 \quad \# 2$ & 2 \\
\hline \multicolumn{2}{r|}{ Total weighted value } & $6 \leq 7$ \\
\hline
\end{tabular}

By comparing the planning for the two different Epsilon values, it can be concluded that the optimal routing and scheduling can be affected severely by changing the Epsilon value.

Nurse $\# 1$ visited patients 10, 6, 3, 5 and 7 in the second planning instead of patients 8,9 and 5 . The optimal planning for nurse $\# 2$ is proposes to serve patient 9 instead of patients 6,2 and 7 . Nurse \#3 visited patients 8 and 2 in the second planning instead of patients 10 and 3.

As it can be understood from the results (Figure 3 and Figure 4)), although decreasing of Epsilon parameter can reduce the downgrading costs of the company, the summation of traveling times of the nurses will be increased from 600.43 to 639.761, and the company will have larger operational costs. So in this context, the decision maker should make a tradeoff between reducing the downgrading costs and increasing the total travelling times of the nurses. 


\subsection{Results}

In this subsection, three different categories of instances are tested to show the effectiveness of the proposed model. The properties of the benchmark instances are described in Table 6 .

Table 6: Properties of the benchmark instances

\begin{tabular}{ccccc}
\hline Category & $\begin{array}{c}\text { Instance } \\
\text { number }\end{array}$ & $\begin{array}{c}\text { Number of } \\
\text { patients }\end{array}$ & $\begin{array}{c}\text { Number of } \\
\text { nurses }\end{array}$ & $\begin{array}{c}\text { Number of } \\
\text { services }\end{array}$ \\
\hline 1 & $\# 1-\# 10$ & 10 & 3 & 6 \\
2 & $\# 11-\# 20$ & 25 & 5 & 6 \\
\hline
\end{tabular}

To the best of the authors' knowledge, this study is the first one which considers downgrading costs in the routing and scheduling of the home health care problem. Accordingly, the value of the parameter $\mathrm{w}_{\mathrm{s}}$ is settled by the authors. The supposed weighted values of different service types are presented in Table 2.

The obtained solutions for different categorizes of problem instances are given in Table 7 and Table 8, respectively.

Table 7: Solutions for the instances of the first category

\begin{tabular}{c|c|c}
\hline Instance number & Epsilon parameter & Optimal solution value \\
\hline$\# 1$ & 10 & 600.43 \\
\hline$\# 2$ & 10 & 426.722 \\
\hline$\# 3$ & 10 & 602.677 \\
\hline$\# 4$ & 10 & 519.302 \\
\hline$\# 5$ & 10 & 681.19 \\
\hline$\# 6$ & 10 & 475.042 \\
\hline$\# 7$ & 10 & 357.028 \\
\hline$\# 8$ & 10 & 387.626 \\
\hline$\# 9$ & 10 & 583.52 \\
\hline$\# 10$ & 10 & 677.085 \\
\hline
\end{tabular}

Table 8: Solutions for the instances of the second category

\begin{tabular}{c|c|c}
\hline Instance number & Epsilon parameter & Optimal solution value \\
\hline$\# 11$ & 20 & 904.743 \\
\hline$\# 12$ & 20 & 823.3 \\
\hline$\# 13$ & 20 & 765.121 \\
\hline$\# 14$ & 20 & 904.989 \\
\hline$\# 15$ & 20 & 1833.752 \\
\hline$\# 16$ & 20 & 825.067 \\
\hline$\# 17$ & 20 & 626.793 \\
\hline$\# 18$ & 20 & 705.303 \\
\hline$\# 19$ & 20 & 1115.815 \\
\hline$\# 20$ & 20 & 432.561 \\
\hline
\end{tabular}


According to the obtained results, one can conclude that the proposed bi-objective model in this paper can be well used for various daily planning of home health care organizations in different sizes and can help them in their routing and scheduling decisions as well.

\subsection{Sensitivity Analysis}

\subsubsection{Effect of the Epsilon parameter on the optimal solution value}

In this section, a sensitivity analysis on the Epsilon parameter of the model is performed to get a better insight into the effects of changing downgrading decisions on the whole model results. The chosen parameter for the sensitivity analysis is $\varepsilon$ which determines to which amount the decision maker is ready not to use his/her precious human resources capabilities. In fact, Epsilon is the difference between the potential skills of the nurses of the company and the actually used skills in the routing and scheduling process. The results of the sensitivity analysis on the Epsilon parameter for the instance with 25 patients, 5 nurses and 6 service types are presented in Table 9 .

Table 9: A sensitivity analysis of the results on the parameter Epsilon

\begin{tabular}{c|c|c}
\hline Row number & Epsilon parameter & Optimal solution value \\
\hline$\# 1$ & 9 & 1114.781 \\
\hline$\# 2$ & 10 & 1007.037 \\
\hline$\# 3$ & 12 & 924.855 \\
\hline$\# 4$ & 13 & 924.855 \\
\hline$\# 5$ & 15 & 917.103 \\
\hline$\# 6$ & 18 & 904.743 \\
\hline$\# 7$ & 20 & 904.743 \\
\hline
\end{tabular}

The effect of changing the Epsilon parameter on the final result is illustrated in Figure 5.

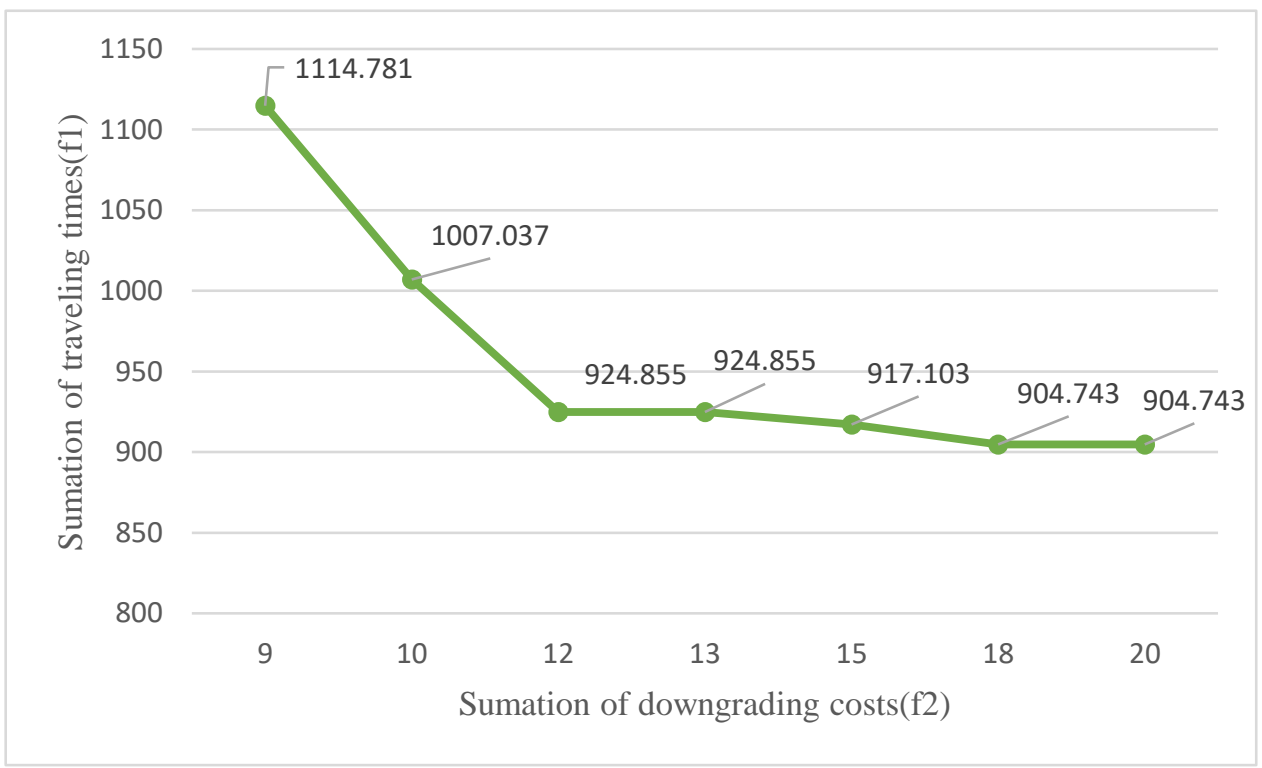

Figure 5: A sensitivity analysis of the results on the parameter Epsilon 
From Figure 5, it can be seen that the smallest Epsilon value could be 9, and the model is infeasible for lower values. In addition, by making the Epsilon value larger than 18, the final optimal solution does not change, and 18 is an upper bound for this problem.

As it is obvious from Figure 5, the optimal solution is affected when changing the Epsilon parameter of the bi-objective model. When the downgrading costs are more important for the decision maker, she/he reduces the Epsilon parameter to lessen the dissatisfaction of high-qualified nurses and hidden costs of company. On the other hand, there will be incurred more nurse traveling time costs to the company which is a very important aspect of home health care operational costs. In fact, the decision maker should make a careful tradeoff between reducing downgrading costs and increasing total traveling time costs.

\subsubsection{Upper limit for the meaningful Epsilon parameter}

According to the results of Section 4.3.1, it can be inferred that, if the decision maker is willing to incur more downgrading cost, the optimal solution of the main objective function will be reduced. This section addresses the question how much increase in the downgrading cost will continue to improve the optimal solution. An instance with 25 patients, 5 nurses and 6 service types is used to investigate this issue. The effect of an increase of the downgrading cost on the objective function of problem is demonstrated in Table 10.

Table 10: Actually used downgrading cost

\begin{tabular}{c|c|c}
\hline Row number & Epsilon parameter & Actually used downgrading cost \\
\hline$\# 1$ & 10 & 10 \\
\hline$\# 2$ & 12 & 12 \\
\hline$\# 3$ & 13 & 13 \\
\hline$\# 4$ & 15 & 15 \\
\hline$\# 5$ & 18 & 18 \\
\hline$\# 6$ & 20 & 18 \\
\hline$\# 7$ & 25 & 18 \\
\hline$\# 8$ & 30 & 18 \\
\hline$\# 9$ & 40 & 18 \\
\hline$\# 10$ & 50 & 18 \\
\hline
\end{tabular}

As one can see from Table 10, it can be concluded that, if the Epsilon parameter is increased to values greater than 18, there will be no effect on the total points of the difference between the potential and used qualifications of the nurses, and this violation will always remain equal to 18 . This indicates that the optimal solution will not always improve as the downgrading cost increases, and there is an upper limit for the maximum amount of Epsilon which can be considered. Therefore, if the decision maker increases the Epsilon parameter to upper values, she/he will no longer see a change in the optimal solution because the model does not require a greater amount of mismatch between the potential and used qualifications of the nurses and has reached an optimal solution.

\section{Managerial insights}

Some important managerial insights can be extracted from this study as follows:

1. This novel mathematical model could be used by managers for a better planning of the company's nurses considering downgrading aspects. The managers could make an appropriate 
tradeoff between downgrading and total traveling times of the nurses. The downgrading level could be adjusted by changing the Epsilon parameter of the model.

2. In most of traditional home health care routing and scheduling models, the home health care decision maker assumes that each patient requires only one type of service. In fact, if a patient needs three services simultaneously, the plan considers it as three different patients who have the same place and health profile. The managers of the health care industry can decrease the volume of data in their companies by using this new method as well. Actually, in this plan each patient has a unique physical and heath profile, where besides removing multiple same profiles for each patient, the home health care company can have a clean and rich database from their customers. Also, top level managers can use this valuable resource to establish marketing strategies or manage their employees and service capacities.

3. Downgrading concepts could help the managers for making better nurse capability decisions. The managers could understand that there are some unrequired nurse qualifications in their company or there are extra needs for new skills and he/she should hire additional skillful nurses for getting a better service level to the patients.

\section{Conclusions and future studies}

Health care has always been a vital concern of humans throughout history. Therefore, human societies have always tried to improve their health. Governments nowadays spend a significant portion of their budget on health. Therefore, an optimization in this field has been of great interest to researchers in recent years. In general, researchers have conflicting goals in this optimization. In addition to reducing the costs, they should increase the quality of the provided services to ensure the maximum stakeholders' satisfaction.

Due to the limited resources available in health systems nowadays, there are many concerns about providing appropriate services to patients. The capacity of hospital beds does not meet the needs of patients, and hospital admission departments are always crowded. One of the most recent ways of providing services to patients is the provision of appropriate services to the patients at their home. Home health care can reduce unnecessary hospital admissions and make patients more comfortable. Moreover, nosocomial infections are one of the most important issues with the hospitalization of patients which always cause many problems for the patients. These infections will be reduced by providing services to patients at home. Therefore, applying home health care in addition to reducing costs will also improve the process of providing services to patients.

One of the most important goals that have always been considered in the field of home health care is to increase the level of stakeholders' satisfaction. Nurses are one of the most important stakeholders in this problem. Not using some of the nurses' skills may lead to a dissatisfaction of the nurses. Despite the nurses' dissatisfaction, it would incur a hidden cost to the service provider because the plan did not use all of the potential available resources that are very valuable to the home health care company.

In this study, a bi-objective model was proposed to minimize the downgrading costs which characterize the difference between the potential and actual skills of the nurses as well as to minimize the total traveling time of the nurses. In order to solve the proposed bi-objective model, an Epsilon-constraint based solution approach was developed. In the first section of the computational experiments, the importance of the model was discussed through interpreting the obtained results obtained by solving a small example, and the applicability of the proposed model was shown. Then the model was applied to several sets of problems including different sizes to confirm the efficiency of the new model for various home health care problems. Moreover, to analyze the effect of the parameter of the solution method on the problem, a sensitivity analysis was conducted on the Epsilon parameter. Finally, some managerial insights for health care managers were presented to help them to well handle their available resources.

As a direction for future research, the application of heuristic and meta-heuristic algorithms to solve larger-sized instances could be useful (Kolahan \& Kayvanfar, 2009; Kayvanfar et al., 2015), 
specifically when exact approaches cannot be developed or are inefficient. It would be interesting to apply the proposed model to uncertain situations. Another direction could be the use of exact techniques like the branch and cut $(B \& C)$ method to solve the proposed model. Since this type of problem could be evaluated from different perspectives, owing to existing different stakeholders' goals and proposals, adding various goals to the model could be useful. Different multi-objective algorithms could be applied to the model to compare the solutions obtained. Finally, developing the model using time-dependent travel times in urban regions could be another stream. 


\section{References}

1. A. A. Alodhayani, "Comparison between home health care and hospital services in elder population: cost-effectiveness," Biomedical Research, 2017.

2. M. Tyan, "Understanding Taiwanese home healthcare nurse managers' empowerment and international learning experiences: Community-based participatory research approach using a US home healthcare learning tour," ProQuest Dissertations Publishing, Washington, 2010 .

3. A. Fernandez, G. Gregory, A. Hindle, and A. Lee, "A model for community nursing in a rural county," Journal of the Operational Research Society, vol. 25, no. 2, pp. 231-239, 1974.

4. S. Bertels and T. Fahle, "A hybrid setup for a hybrid scenario: combining heuristics for the home health care problem," Computers \& Operations Research, vol. 33 ,no. 10, pp. 2866-2890, 2006.

5. P. Eveborn, P. Flisberg, and M. Rönnqvist, "Laps Care - an operational system for staff planning of home care," European journal of operational research, vol. 171, no. 3, pp. 962-976, 2006.

6. C. Akjiratikarl, P. Yenradee ,and P. R. Drake, "PSO-based algorithm for home care worker scheduling in the UK," Computers \& Industrial Engineering, vol. 53, no. 4, pp. 559-583, 2007.

7. A. Trautsamwieser, M. Gronalt, and P. Hirsch, "Securing home health care in times of natural disasters," OR spectrum, vol. 33, no. 3, pp. 787-813, 2011.

8. A. Trautsamwieser and P. Hirsch, "Optimization of daily scheduling for home health care services," Journal of applied operational research, vol. 3, no. 3, pp. 124-136, 2011.

9. D. S. Mankowska ,F. Meisel, and C. Bierwirth, "The home health care routing and scheduling problem with interdependent services," Health care management science, vol. 17, no. 1, pp. 15-30, 2014. [Online]. Available: https://link.springer.com/article/10.1007\%2Fs10729-013.9243-1-

10. M. Misir, P. Smet, and G. Vanden Berghe, "An analysis of generalised heuristics for vehicle routing and personnel rostering problems," Journal of the Operational Research Society, vol. 66, no. 5, pp. 858-870, 2015.

11. B. Yuan, R. Liu, and Z .Jiang, "A branch-and-price algorithm for the home health care scheduling and routing problem with stochastic service times and skill requirements," International Journal of Production Research, vol. 53, no. 24, pp. 7450-7464, 2015.

12. K. Braekers, R .F. Hartl, S. N. Parragh, and F. Tricoire, "A bi-objective home care scheduling problem: Analyzing the trade-off between costs and client inconvenience," European Journal of Operational Research, vol. 248, no. 2, pp. 428-443, 2016.

13. A. Dohn, E. Kolind, and J. Clausen, "The manpower allocation problem with time windows and job-teaming constraints: A branch-and-price approach," Computers \& Operations Research, vol. 36, no. 4, pp. $1145-1157,2009$.

14. H. Allaoua, S. Borne, L. Létocart, and R. W. Calvo" ,A matheuristic approach for solving a home health care problem," Electronic Notes in Discrete Mathematics, vol. 41, pp. 471-478, 2013.

15. G. Hiermann, M. Prandtstetter, A. Rendl, J. Puchinger, and G. R. Raidl, "Metaheuristics for solving a multimodal home-healthcare scheduling problem," Central European Journal of Operations Research, vol. 23, no. 1, pp. 89-113, 2015.

16. M. Mutingi and C. Mbohwa, "Multi-objective homecare worker scheduling: A fuzzy simulated evolution algorithm approach," IIE Transactions on Healthcare Systems Engineering, vol. 4, no. 4, pp. 209-216, 2014.

17. K.-D. Rest and P. Hirsch, "Daily scheduling of home health care services using time-dependent public transport," Flexible Services and Manufacturing Journal, vol. 28, no. 3, pp.2016,495-525 .

18. C. Fikar and P. Hirsch, "A matheuristic for routing real-world home service transport systems facilitating walking," Journal of Cleaner Production, vol. 105, pp. 300-310, 2015.

19. P. Toth and D. Vigo, Vehicle routing: problems, methods, and applications. SIAM, 2014.

20. R. Redjem and E. Marcon, "Operations management in the home care services: a heuristic for the caregivers' routing problem," Flexible Services and Manufacturing Journal, vol. 28, no. 1-2, pp. 280-303, 2016.

21. C .Rodriguez, T. Garaix, X. Xie, and V. Augusto, "Staff dimensioning in homecare services with uncertain demands," International Journal of Production Research, vol. 53, no. 24, pp. 7396-7410, 2015.

22. R. Liu, B. Yuan, and Z. Jiang, "Mathematical model and exact algorithm for the home care worker scheduling and routing problem with lunch break requirements," International Journal of Production Research, vol. 55, no. 2, pp. 558-575, 2017.

23. B. Yuan, R. Liu, and Z. Jiang, "Daily scheduling of caregivers with stochastic times," International Journal of Production Research, vol. 56, no. 9, pp. 3245-3261, 2018. 
24. M. Liu, D. Yang, Q. Su, and L. Xu, "Bi-objective approaches for home healthcare medical team planning and scheduling problem," Computational and Applied Mathematics, vol. 37, no. 4, pp. 4443-4474, 2018.

25. J. Decerle, O. Grunder, A. H. El Hassani, and O. Barakat, "memetic-ant colony optimization algorithm for the home health care problem with time window, synchronization and working balancing ",Swarm and Evolutionary Computation, January 2019.

26. J. Nasir and C. Dang, "Solving a more flexible home health care scheduling and routing problem with joint patient and nursing staff selection," Sustainability, vol. 10, no. 1, p. 148, 2018.

27. J. A. Nasir, S. Hussain , and C. Dang, "An integrated planning approach towards home health care, telehealth and patients group based care," Journal of Network and Computer Applications, vol. 117, pp. 30-41, 2018.

28. A. M. Fathollahi-Fard, M. Hajiaghaei-Keshteli, and R. Tavakkoli-Moghaddam, "A bi-objective green home health care routing problem," Journal of Cleaner Production, vol. 200, pp. 423-443, 2018.

29. M .Laumanns, L. Thiele, and E. Zitzler, "An efficient, adaptive parameter variation scheme for metaheuristics based on the epsilon-constraint method," European Journal of Operational Research, vol. 169, no. 3, pp. 932-942, 2006.

30. F. Kolahan, V. Kayvanfar, "A heuristic algorithm approach for scheduling of multi-criteria unrelated parallel machines", In proceeding of International Conference on Industrial and Mechanical EngineeringICIME09 (World Academy of Science, Engineering and Technology, WASET), Vol. 59, pp. 102-105, 2009.

31. V. Kayvanfar, E. Teymourian and K. M. Alizadeh, "Intelligent water drops algorithm on parallel machines scheduling," 2015 International Conference on Industrial Engineering and Operations Management (IEOM), Dubai, 2015, pp. 1-5, doi: 10.1109/IEOM.2015.7093842. 